\title{
Desafíos actuales en torno a la investigación en apego infantil en el contexto chileno ${ }^{1}$
}

\section{Current challenges around research in infant attachment in the Chilean context}

\author{
Marcela Aracena², María Pía Santelices, Chamarrita Farkas, Maribel González, Ema Fugellie \\ Escuela de Psicología, Pontificia Universidad Católica de Chile \\ Mónica Guzmán \\ Escuela de Psicología, Pontificia Universidad Católica de Chile. \\ Escuela de Psicología, Universidad Católica del Norte
}

(Recepción: Septiembre 2008 - Aceptación: Noviembre 2008)

\begin{abstract}
Resumen
En aras de generar conocimiento contextualizado con los recursos disponibles, el presente artículo hace una revisión de aquellos aspectos necesarios de considerar en torno a la investigación en apego infantil en Chile. La revisión se centra en tópicos agrupados en características de la población a intervenir, características de las intervenciones, y aspectos metodológicos. Por otra parte se presenta la complejidad del contexto chileno, mostrando las fortalezas y dificultades encontradas para lograr los estándares de calidad internacionales en investigaciones en apego. Se concluye que para diseñar programas de intervención temprana en apego que sean efectivos, es necesario tomar en cuenta, entre otros, factores tales como: el foco de la intervención, variables mediadoras; evaluaciones a corto y largo plazo; inclusión de un tamaño muestral que permita extraer conclusiones significativas y soportar las pérdidas, y utilizar instrumentos válidos y confiables. Por otra parte se postula la necesidad de unir esfuerzos tanto intelectuales como económicos.

Palabras Clave: Apego, Diseño de investigación, Intervenciones tempranas.
\end{abstract}

\begin{abstract}
In order to generate knowledge with the available resources, this article reviews the necessary aspects to be considered around research in infant attachment in Chile. Its focuses at topics grouped around population characteristics to be intervened, interventional characteristics and methodological aspects. It also acknowledges the complexity of the Chilean context, showing the strengths and difficulties met in achieving the international quality standards for mother-child attachment research. One concludes that to design programs of early intervention in mother-child attachment that are effective, it is necessary to take into consideration, between others, factors, such like: the focus of the intervention; mediation variables; short and long term evaluations, inclusion of an adequate sample size which allows obtaining significant conclusions, have a proper drop-out rate, and the use of valid and trustful instruments. On the other hand, there is postulated the need to join both intellectual and economic efforts.
\end{abstract}

Key Words: mother-child attachment, research design, early interventions.

1 El presente artículo contó con el financiamiento del Proyecto FONDECYT 1040760.

2 Correspondencia: Marcela Aracena, Av. Vicuña Mackenna 4860, Santiago de Chile. Teléfono: 3545915. Correo electrónico: maracena@uc.cl 


\section{Introducción}

Quien diseña una política de intervención temprana debe tomar en cuenta factores diferenciales que puedan afectar la efectividad de una intervención (Strasser, 2006), afirmación que se torna especialmente relevante cuando los resultados de una intervención en apego no se comportan de acuerdo a lo esperado.

¿Qué dice la literatura al respecto? Strasser (2006) realiza una síntesis de aquellos aspectos que deben ser tomados en cuenta a la hora de evaluar programas específicos. Señala que factores tales como el nivel de riesgo, las habilidades de entrada, el contexto familiar, y la edad de los participantes, pueden modificar los resultados de efectividad de un programa de promoción y prevención, en este caso, en apego seguro. La autora plantea que ignorar estas interacciones puede llevar a los investigadores a conclusiones erróneas acerca del efecto de la intervención.

Flay, Biglan, Boruch, Castro, Gottfredson, Kellam, et al. (2005) (citado en Olds, 2007), por su parte, han sugerido distintos estadios de la investigación en intervenciones parentales, como asimismo, una serie de estándares para que una investigación sea considerada de calidad. Entre ellas se plantea que es necesario tener una clara operacionalización de la intervención, que se deben usar diseños rigurosos, así como clarificar la muestra, utilizar variables resultados válidas, utilizar métodos estadísticos apropiados, considerar el valor práctico que tiene la intervención a nivel de políticas públicas, además de tener información y réplica de al menos dos estudios independientes que repliquen el programa. Asociado a lo anterior, para que una intervención sea considerada efectiva, debe cumplir otros estándares de calidad, entre ellos, un programa operacionalizado en un manual con entrenamiento y soporte técnico, una teorización de los mecanismos causales, claridad acerca de la población beneficiada, mediciones acerca de la exposición de la intervención, de la integridad y de la implementación, y métodos de muestreo adecuados para captar la población objetivo. También se debe conocer el valor práctico de la intervención propuesta e hipótesis incluyendo la población que probablemente se beneficie.

Por otra parte, y en relación a investigaciones específicamente en el campo del apego, las revisiones meta-analíticas realizadas por Bakermans-Kranenburg, Juffer y van Izjerdoorn (2003) y Bakermans-Kranenburg, van Izjerdoorn y Juffer (2005), muestran la importancia de considerar variables mediadoras, tales como la sensibilidad materna en la evaluación de la eficacia de los programas, dado que los efectos en el corto y mediano plazo se harían más evidentes en dichas variables que en el apego propiamente tal. Además, se menciona que características del programa mismo, tales como su duración, tendrían influencia sobre la efectividad (intervenciones más breves serían más efectivas). Adicionalmente, ambas revisiones coinciden en mencionar que mientras más porcentaje de apego desorganizado exista en el grupo control, mayor sería la efectividad del programa.

A partir de los antecedentes anteriores, y teniendo como fundamento la necesidad de diseñar intervenciones que permitan extraer conclusiones válidas de dichas propuestas de investigación, el presente artículo pretende sistematizar los diversos aspectos que habría que considerar al momento de diseñar investigaciones en promoción y/o prevención en el ámbito del apego, a partir de los aportes encontrados en la literatura respecto del tema, como asimismo hacer una reflexión acerca de las principales dificultades que se presentan en el contexto chileno para llevar a cabo investigaciones que cumplan con estos estándares. Al respecto, los factores a considerar pueden ser organizados en torno a 3 grandes tópicos, tal como puede apreciarse en la Figura 1. 


\section{Figura 1}

Variables a considerar en el diseño de programas de investigación tendientes a evaluar la efectividad de programas de intervención temprana en apego

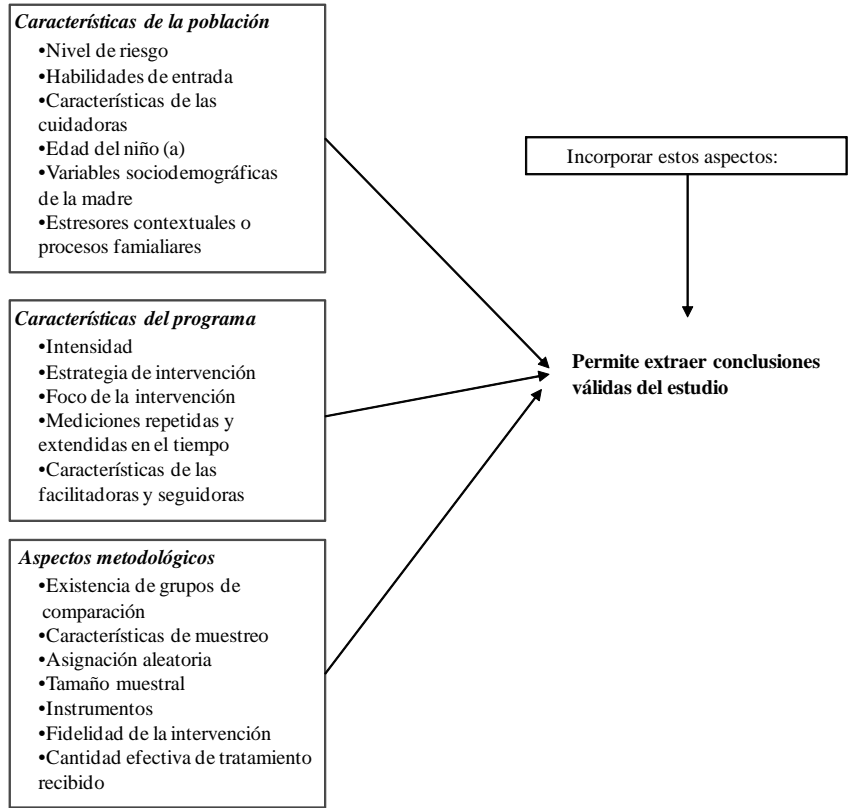

Tal como lo indica el diagrama, un primer aspecto es reconocer para quién o quiénes la intervención podría ser eficaz. Un segundo aspecto guardaría relación con las características que ha de tener el programa mismo. Finalmente, un tercer aspecto se refiere a las características metodológicas y de diseño de los programas de intervención, de modo tal que permitan evaluar la validez de los resultados obtenidos. Estos elementos son los que se revisan en mayor profundidad a continuación.

\section{Características de la Población Objetivo}

Aun cuando un programa de intervención puede resultar efectivo, es necesario considerar que esa efectividad se expresa de diferentes maneras en distintos grupos de estudio, es decir, depende de las características de la población objetivo.

Dentro de este ámbito, un primer factor guarda relación con el nivel de riesgo de la población a intervenir, definido en términos de pobreza y/o falta de apoyo social (Ansfield et al., 1990; Heinicke et al., 2001). Ello porque trabajar con poblaciones con distintos niveles de riesgo puede tener efectos diversos. Por ejemplo, el meta análisis realizado por Bakermans-Kranenburg, et al. (2005), señala que, de acuerdo al nivel de riesgo en apego, los resultados podrían ser diferenciales, de modo tal que intervenciones tempranas muestran resultados divergentes en apego desorganizado, desde efectos positivos a negativos. Específicamente, se reporta que la efectividad de la intervención aumenta mientras mayor es el nivel de desorganización en el apego de la madre al inicio del estudio (Bakermans-Kranenburg et al., 2003).

Desde otra perspectiva, intervenciones dirigidas a madres que presentaban un diagnóstico clínico de depresión postparto, mostraron efectos pequeños en reducir apego desorganizado (Gelfland, Teti, Seiner \& Jameson, 1996; Cooper \& Murray, 1997).

Por otro lado, intervenciones que combinan distintos tipos de riesgo psicosocial, tales como: pobreza, bajo apoyo social y parentalidad inadecuada, han tenido efectos positivos en aumentar 
la sensibilidad materna y el respeto de la madre por la autonomía del niño/a, mejorar los patrones normativos de apego, y reducir el apego desorganizado (Heinicke, et al., 1999).

En suma, y de acuerdo a lo que señala Beckwith (2000 citado en Ammaniti et al., 2006), pareciera ser que existe una asociación en forma de U invertida entre el número de factores de riesgo y la eficacia de la intervención, de modo tal que en muestras con muy pocos factores de riesgo, los efectos de la intervención pueden ser menos claros, de manera que madres con buen funcionamiento inicial se beneficiarían menos del programa.

Este último punto guarda estrecha relación con un segundo factor a considerar: las habilidades de entrada de los sujetos respecto a los aspectos a entrenar, las cuales pueden explicar la diversidad del tamaño de los efectos de las intervenciones. Así por ejemplo, resulta más complejo obtener resultados significativos desde un punto de vista estadístico cuando las madres a intervenir son ya lo suficientemente buenas en las habilidades a entrenar o han tenido intervenciones previas en habilidades similares. Madres con patrones de apego seguro es probable que sean madres suficientemente buenas aun sin intervención (Ammaniti et al., 2006). Estos dos aspectos hacen que el desarrollo de programas de promoción de apego seguro en población normal (con bajo riesgo y buenas habilidades de entrada) implique un desafío adicional.

Dentro de las habilidades de entrada, una de las variables más estudiadas ha sido la sensibilidad de la madre o cuidador para responder al niño/a (Sajaniemi, Makela \& Salokorpi, 2001; Anisfeld, Casper, Nocyze \& Cunningham, 1990), habiéndose constituido en uno de los principales focos de intervención. Referente a esto, se ha demostrado que es posible mostrar diferencias significativas en el nivel de sensibilidad de las madres post intervención (Anisfeld et al., 1990; van den Boom, 1994; Bakermans-Kranenburg, et al., 1998; Juffer et al., 2005; Velderman, Bakermans-Kranenburg, Juffer \& van Izjerdoorn, 2006).

Strasser (2006) plantea que un tercer grupo de variables que también podría contribuir a explicar el éxito o fracaso de las intervenciones son las características de las cuidadoras, incluyendo entre ellas el nivel educacional y la edad de éstas.

Específicamente, estudios empíricos indican diferencias significativas en los resultados de las intervenciones según el nivel educacional de las cuidadoras, de modo tal que cuidadoras de tipo profesional tendrían más efectos en la línea cognitiva, mientras que las de formación no profesional los tendrían más a nivel emocional (Olds, et al., 2002; Olds, et al., 2004).

Respecto a la edad de la madre, se ha mencionado que existe un mayor riesgo cuando éstas son adolescentes (Organización de las Naciones Unidas [ONU], 2000; Baranowsky, Schillmoller \& Higgins, 1990, citado en Ortiz, Borré, Carrillo \& Gutiérrez, 2006).

Los resultados muestran que la edad del niño/a al inicio de intervenciones en apego, se asocia significativamente con el grado de efecto, instalando la cuestión referente a la interacción entre la intervención y la edad de los sujetos participantes. En apego los resultados al respecto son contradictorios.

El discurso de "ventanas de oportunidad” es una hipótesis que aún no cuenta con todo el respaldo en la literatura (Strasser, 2006). Algunos clínicos sostienen que el sistema madre-hijo/a al momento del nacimiento se encuentra más abierto a recibir apoyo social, consejos e intervenciones (Olds, Henderson, Chamberlin, \& Tatelbaum, 1986; Crnic \& Greenberg, 1987). Sin embargo, BakermansKranenburg et. al. (2003) no apoyan esta afirmación; ellos postulan que el momento del nacimiento genera altos niveles de estrés en los padres, debido a que éstos deben adaptarse a una nueva situación familiar y a las demandas de cuidado del niño/a. Por otro lado, existen casos clínicos particulares en los cuales es necesario apoyar a las madres a los pocos meses de nacimiento del niño/a, como por ejemplo, cuando la madre presenta depresión postparto (Cooper \& Murray, 1997). Otras investigaciones sugieren que es necesario comenzar las intervenciones en el período prenatal, principalmente en el caso de trabajar con población de alto riesgo, ya que de esta manera se genera el apoyo social necesario para que los cuidadores aprendan modelos de control no coercitivos durante la crianza del niño/a (Egeland \& Ericsson, 1993; Heinicke et al., 1999). 
Una visión más global respecto al tema la entrega la revisión meta-analítica realizada por Bakermans-Kranenburg et al. (2003), que muestra que los programas que comienzan después de los primeros seis meses de vida del niño/a, son más efectivos que aquellos que comienzan en el período prenatal.

Otro aspecto relevante de ser considerado tanto en el diseño como en la evaluación del éxito o fracaso de una intervención se relaciona con las variables sociodemográficas (Belsky, 1984/1990, citado en Cassidy \& Shaver, 1999). En intervenciones en apego, se recomienda relacionar múltiples factores de riesgo de manera simultánea para poder establecer una relación más clara con los patrones de apego (Belsky, 1999, citado en Huth-Bocks, Levendosky, Bogat \& Von Eye, 2004).

Finalmente, se deben tomar en cuenta en la evaluación de intervenciones tempranas, los estresores contextuales o procesos familiares que viven las personas que participan del programa, tales como: nivel socioeconómico bajo, monoparentalidad y violencia, que se relacionan preferentemente con un estilo de apego inseguro del niño/a (Belsky, 1999, citado en Huth-Bocks, Levendosky, Bogat \& Von Eye, 2004).

Además, Heinicke, Diskin, Ramsey-Klee y Oates (1986), reportan, a partir de estudios longitudinales, que el apoyo paterno y de la familia son fundamentales para explicar diferencias en los resultados de una intervención en apego con población en riesgo. Madres con altos niveles de estrés tienden a tener menos actitudes positivas al interactuar con sus bebés. El apoyo social tiende a moderar las respuestas ante el estrés, y favorecer una actitud positiva frente a las necesidades del niño/a (Crittenden, 1985; Jacobson \& Frye, 1991).

\section{Características del Programa de Intervención}

Un primer aspecto respecto a las características del programa mismo, guarda relación con la intensidad óptima de éste, es decir, el número de horas, días y semanas de la intervención. La mayoría de los programas de intervención temprana que han mostrado efectos importantes y duraderos han sido muy intensos (Ammaniti et al., 2006). Sin embargo, este tema también está abierto a discusión, ya que algunos autores mencionan que las intervenciones de reducido número de sesiones son más efectivas, tal como ocurre con las revisiones meta-analíticas antes mencionadas (Bakermans-Kranenburg et al., 2003; Bakermans-Kranenburg, et al., 2005).

$\mathrm{Al}$ respecto, y a fin de evaluar estos programas, Gurung (2006) recomienda no sólo incluir el grupo experimental y de control, sino además, grupos de intensidades intermedias para poder estudiar cuál es la condición óptima para que el programa sea efectivo.

Un segundo aspecto guarda relación con la estrategia de intervención utilizada. Al respecto, éstas han sido variadas y los estudios no son concluyentes respecto a cuál de ellas es la más eficaz. Algunas trabajan desde el embarazo hasta el primer año de vida, utilizando como estrategia las visitas domiciliarias (Jacobson \& Frye, 1991; Huxley \& Warner, 1993; Heinicke et. al., 1999; Ammaniti et al., 2006). Otras intervenciones en cambio, han mostrado efectos positivos combinando distintas estrategias, tales como; visitas domiciliarias, video feedback y apoyo psicoeducacional (Juffer, Bakermans-Kranenburg \& van Izendoorn, 2005; Velderman, et al. 2006).

Desde otra perspectiva, otro aspecto importante a considerar se relaciona con el modelo o las teorías que subyacen a ésta y la función de variables mediadoras que podrían constituirse en el foco de intervención. Una variable ejerce un efecto de mediación cuando ella representa el mecanismo generativo a través del cual la variable independiente (en este caso un programa de intervención temprana) ejerce sobre la dependiente (Strasser, 2006). En apego, existen distintas teorías que permiten explicar los cambios. Algunas apuntan a que es necesario tener como foco las representaciones mentales, mientras que otras proponen trabajar sobre la sensibilidad de los padres (Bakermans-Kranenburg, et al., 2005). Respecto a esta última variable, cabe mencionar que si bien existe apoyo a la noción de que la sensibilidad explica una proporción de la varianza en apego, lo que justifica incluirla como un foco de intervención, es importante considerar que su contribución al 
apego sigue siendo pequeña, y que los mecanismos que subyacen a la transmisión intergeneracional del apego son aún materia de estudio, lo que se ha denominado transmission gap (van Izendoorn, 1995; Madigan et al., 2006).

No obstante lo anterior, la mayoría mide como variable resultado sólo el estilo de apego y no variables mediadoras que pueden constituirse en el foco de los programas de intervención.

Además, en la evaluación de los programas de intervención en apego suelen realizarse evaluaciones a corto plazo (generalmente centradas sólo en el apego), y no a mediano o largo plazo en otras variables que pueden mostrar cambios, tales como el desarrollo psicomotor de los niños/as (Heinicke, Beckwith \& Thompson, 1988; Heinicke et al., 1999; Ammaniti et al., 2006).

La evaluación de una intervención temprana debería incorporar elementos cognitivos, lingüísticos y socio-emocionales, aunque su interés sea sólo uno de ellos (Strasser, 2006). En intervenciones en este campo, una de las problemáticas en evaluación ha sido que preferentemente se ha medido la variable apego (Juffer, Bakermans-Kranenburg \& van Ijzendoorn, 2005). Lo anterior podría producir error en la interpretación de los resultados. Por ejemplo, podría darse que no se encontraran diferencias significativas en apego, pero sí en un mediano plazo en términos de desarrollo integral (Cooper \& Murray, 1997; Sajaniemi, Makela \& Salokorpi, 2001).

Esta cuestión instala otro tema respecto a los programas en apego: la duración de los efectos de una intervención. Al respecto, teorías del desarrollo humano atribuyen un rol influyente en las etapas tempranas. No obstante, la evidencia científica empírica no logra sostener esta afirmación, especialmente cuando las intervenciones son esporádicas y sin continuidad (Strasser, 2006; Gurung, 2006).

Existe controversia sobre el significado de un efecto inmediato que luego desaparece en el tiempo. Este desvanecimiento puede reflejar no una falta de eficacia de la intervención, sino más bien, el efecto sostenido de otros estímulos negativos después de que ésta termina. Esto puede suceder cuando las madres egresan de una intervención y se insertan en ambientes pobres. Ramey y Ramey (2006) han propuesto el principio de la continuidad del apoyo educativo. Este principio propone que los efectos de una intervención disminuyen con el paso del tiempo si no existen apoyos educativos que mantengan las actitudes y conductas aprendidas. Un programa que no es capaz de mostrar efectos de largo plazo, no puede adjudicarse que es efectivo, ni eficaz (Strasser, 2006). Varios programas con foco en el apego, sólo han realizado mediciones en el corto plazo, preferentemente al primer año de vida (Heinicke et al., 1999; Ammaniti et al., 2006). Sin embargo, otros programas han logrado demostrar efectos positivos a dos años plazo post intervención (Heinicke, Fineman, Ponce \& Guthrie, 2001). Es decir, otros autores dan cuenta de que es posible que los cambios en la interacción madre-hijo/a tengan efectos acumulativos en el largo plazo. Esto debido a que, realizando un seguimiento longitudinal, no logran identificar resultados en el primer año post intervención, pero sí luego de 3 años (a los 4 años de edad del niño/a) (Sajaniemi, Makela \& Salokorpi, 2001). Estos hallazgos ponen de relieve la necesidad de realizar mediciones repetidas y extendidas en el tiempo como otro elemento a considerar dentro de las características del programa mismo.

Finalmente, otro aspecto a considerar en el diseño son las características de las facilitadoras y seguidoras. La literatura señala que a mayor nivel educacional de las personas que realizan la intervención, habría mayor efectividad de ésta en algunas áreas, especialmente en el desarrollo cognitivo (Whitehurst, et al., 1994; Olds, 2000).

\section{Aspectos Metodológicos}

Dentro de los aspectos metodológicos a ser considerados en el momento de evaluar un programa de promoción en apego seguro, se encuentran aspectos del diseño, del muestreo y de los instrumentos a emplear.

Un elemento relevante a la hora de evaluar un programa de intervención en apego dice relación con la existencia de grupos de comparación. Estudios que pretenden mostrar la efectividad de un 
programa de apego han utilizado un grupo control (Bakermans-Kranenburg, et al., 2005).

Otro aspecto que puede producir errores en la interpretación de los resultados de una intervención de promoción en apego se relaciona con las características del muestreo del estudio. Gurung (2006) plantea que si la muestra es heterogénea en variables que podrían interactuar con la intervención (por ejemplo; demográficas, habilidades de entrada y niveles de riesgo), se puede llegar a ocultar efectos significativos cuando sólo se considera el promedio.

Además, la equivalencia inicial de los grupos experimental y control es fundamental para poder afirmar que las diferencias entre los participantes del estudio se deben a la intervención, lo cual se logra idealmente mediante la asignación aleatoria. De hecho, Bakermans-Kranenburg, et al. (2005), sólo incluyen en el meta-análisis los estudios de mayor peso metodológico. Asumen esta decisión afirmando que muchas investigaciones carecen de esta condición, y por tanto, no son relevantes en la investigación en el campo del apego.

Otra consideración relevante para evaluar los efectos de una intervención, es prever la mortalidad experimental. Little (1995, citado en Ahern \& Le Brocque, 2005) señala que todos los estudios experimentan algún grado de deserción, y que éste se incrementa en estudios longitudinales, y en estudios clínicos de medidas repetidas. Las muestras deberían ser lo suficientemente grandes para soportar la pérdida de sujetos y aún así, conservar la potencia suficiente para detectar los efectos de la intervención (Aracena, et al., 2007; Bedregal, 2006; Whitehurst, et al., 1994; Wutzke, Conigrave, Kogler, Saunders, \& Hall, 2000).

Además, un requisito básico para cualquier instrumento es que sea confiable y válido. Los instrumentos utilizados en las investigaciones en apego han sido desarrollados preferentemente en América del Norte o Europa (Ainsworth, Blehar, Waters, \& Wall, 1978; Pierrehumbert, Karmaniola, Sieye, Meister, Miljkovitch \& Halfon, 1996), y se han realizado pocas adaptaciones a nivel local (Santelices, Ramírez, Armijo \& Pérez-Salas, 2008). Por otra parte, los instrumentos están expuestos a muchas fuentes de error, tales como la presencia de otros al momento de la evaluación, y las características del entrevistado/a. El efecto de deseabilidad social ha sido altamente descrito por investigadores en el campo de la medición (Streiner \& Norman, 1999). Existe también la posibilidad de que el evaluador codificador esté sujeto a sesgos producto de su conocimiento sobre la hipótesis del estudio, y de los participantes que pertenecen al grupo control o experimental; en estos casos se debe tener una consideración especial (Streiner \& Norman et al.).

Otro aspecto guarda relación con la fidelidad de la intervención. Strasser (2006) plantea que se debe tener una descripción completa de la intervención antes de implementarla. De este modo, al realizar la evaluación post intervención, se puede constatar si ésta fue fidedigna a su diseño, y relacionar este aspecto con los resultados.

Además, la cantidad efectiva de tratamiento (asistencia) a la que han estado expuestas los/las participantes del grupo de estudio versus el grupo control es otro aspecto que ayuda a identificar el éxito o fracaso de una intervención (Whitehurst et al., 1994).

En resumen, cuando las intervenciones en apego no muestran los efectos esperados, es necesario revisar en forma rigurosa aspectos de diseño del estudio, tales como el empleo de muestras representativas, la administración de instrumentos válidos y confiables, como asimismo, revisar con detención la fidelidad de la intervención (Strasser, 2006).

\section{Complejidad del contexto chileno: Desafíos pendientes}

Para diseñar programas de intervención en apego que sean efectivos, tal como hemos visto en la revisión, es necesario tomar en cuenta entre otros factores; el tipo de foco al cual se apunta, utilizando mediciones a corto y largo plazo, para explicar las diferencias; controlar el tipo de riesgo de la población; incluir un tamaño muestral que permita extraer conclusiones significativas, y soportar las pérdidas; y utilizar instrumentos válidos y confiables, entre otros. La pregunta que queda de fondo es: ¿cuáles son las fortalezas y dificultades reales que experimenta la investigación 
en apego en Chile?

Investigar este tema en el mundo globalizado de hoy en día, es un gran desafío. Si bien en Chile es posible realizar investigaciones que realicen operacionalizaciones claras acerca de las intervenciones en apego, con diseños rigurosos, y métodos estadísticos apropiados, existen algunos tópicos que dificultan realizar un trabajo investigativo que cumpla con todos los estándares establecidos para los ensayos clínicos aleatorizados, lo que se ha denominado internacionalmente los "consort criteria" (Olds, 2007). Los desafíos se presentan en dos niveles: a) La adecuación del concepto de apego al contexto cultural chileno y sus repercusiones en el proceso de evaluación e intervención, y, b) Los recursos para cumplir con los estándares internacionales. Como consecuencia, los productos investigativos obtenidos no necesariamente permiten hacer estudios comparativos con otros países extranjeros.

a) En cuanto al primer punto, un aspecto importante para diseñar intervenciones se relaciona con los modelos o las teorías que explican los procesos involucrados. En apego, existen distintas teorías que permiten dar cuenta de la relación madre o cuidador(a)/bebé. En general, gran parte de los estudios reportados en los últimos meta-análisis de Bakermans-Kranenburg et al. (2003, 2005), son estudios realizados en América del Norte y Europa occidental. Cabe preguntarse si estas aproximaciones teóricas recogen la visión cultural del concepto de apego para aplicarlo a otras culturas, como la nuestra. Una línea de investigación relevante a ser desarrollada apunta a profundizar en el modo de vincularse de los chilenos (modo de expresión del afecto en distintas culturas). Es decir, cómo es la forma de expresar y recibir cariño, el tipo de contacto físico, entre otras.

b) En cuanto a los recursos, estos afectarían a distintos niveles; al nivel de complejidad del diseño, la viabilidad de una muestra representativa, la calidad de los instrumentos seleccionados, el tipo de entrenamiento que reciben los evaluadores para aplicar los instrumentos existentes, entre otras.

\section{Consideraciones acerca del diseño y muestra del estudio}

Estudios que pretenden mostrar la efectividad de un programa de apego han utilizado muestras aleatorizadas con un grupo control, como asimismo, muestras representativas que puedan ser generalizables. ¿Es un problema de diseño de investigación, o un problema de viabilidad económica para llevar a cabo estas investigaciones en Chile? Desde nuestra perspectiva, el tema conceptual y económico son las grandes encrucijadas.

Uno de los desafíos que experimentan las investigaciones en apego en nuestro contexto, es la dificultad para discriminar las características de la muestra y de los grupos de comparación, sin contar, a la fecha, con instrumentos válidos y confiables que permitan distinguir los grupos de entrada al estudio. Además, los equipos de investigación requieren contar con suficientes recursos económicos para que, tanto el grupo muestral, como el grupo de comparación, puedan cumplir con los estándares internacionales de selección de una muestra apropiada.

Otro ámbito que puede producir errores en la interpretación de los resultados se relaciona con las características del muestreo de los estudios. Gurung (2006) plantea que si la muestra es heterogénea en variables que podrían interactuar con la intervención (por ejemplo; demográficas, habilidades de entrada y niveles de riesgo), se puede llegar a ocultar efectos significativos cuando sólo se considera el promedio. Es probable que en estudios chilenos, por razones económicas, sólo se tenga acceso a muestras cautivas, que no necesariamente son representativas, ni diversas, o no se distinga con precisión el tipo de riesgo al cual se enfoca la investigación. También es relevante para evaluar los efectos de una intervención, el prever la mortalidad experimental. Las muestras deberían ser lo suficientemente grandes para soportar la pérdida de sujetos, y aún así, conservar la potencia suficiente para detectar los efectos de la intervención. El tamaño muestral también está en directa relación con los recursos disponibles, al igual que el seguimiento de aquellas personas que no aceptan participar del estudio y de aquellas personas que se retiran en el transcurso de la investigación. Ambos temas son de relevancia para poder extraer conclusiones válidas de las investigaciones. 


\section{Variables e instrumentos}

Un requisito básico para cualquier instrumento es que sea válido y confiable. Los instrumentos utilizados en las investigaciones en apego han sido desarrollados preferentemente en América del Norte o Europa, y se han realizado pocas validaciones a nivel local.

Por otra parte, los instrumentos están expuestos a muchas fuentes de error. El efecto de deseabilidad social ha sido altamente descrito por investigadores en el campo de la medición. Otra de las dificultades en Chile, es que no se dispone de contextos adecuados para la evaluación, generalmente ésta se realiza durante una consulta médica o en los domicilios de los/las participantes. En ambos casos no es posible controlar la presencia de otras personas ajenas al estudio. Lo anterior redunda en una interferencia en el proceso evaluativo global. Por otra parte la mayoría de las muestras utilizadas corresponden a sectores de vulnerabilidad social importante, en las cuales la deseabilidad social está presente en la medida en que se asocia la participación en este tipo de estudios a un eventual mejoramiento de la calidad de atención recibida.

Existe también la posibilidad de que el evaluador codificador esté sujeto a sesgos producto de su conocimiento sobre la hipótesis del estudio, y de los participantes que pertenecen al grupo control o experimental, en estos casos se debe tener una consideración especial. Por razones económicas, en los estudios locales el recurso humano es escaso, y por tanto en ocasiones se utilizan distintas estrategias: a) miembros voluntarios; b) el evaluador realiza otras funciones en el proceso investigativo que pueden interferir en el proceso de evaluación. Por otra parte, en el tema de apego, existen escasos instrumentos validados en Chile, lo cual conlleva a utilizar instrumentos que sólo cuentan con una adaptación preliminar.

Contextualizando el conocimiento existente en nuestro país, se puede señalar que los profesionales de la salud en Chile están interesados en el tema, existiendo diversas iniciativas para fomentar un apego seguro en los bebés, sobre todo durante el embarazo, parto y posteriores controles del bebé (Ossandón, Ilhabaca \& Gajardo, 2000; Rossel, Carreño \& Maldonado, 2002). Respecto a la investigación en Chile acerca del tema del apego, se cuenta con diversas tesis de pre y postgrado que se han llevado a cabo en distintas universidades, en Santiago y regiones, lo que indica el interés creciente en el ámbito académico. Por otro lado, existe un aumento creciente de publicaciones nacionales que presentan resultados de estudios relacionados con el apego, tanto desde un análisis teórico y metodológico (Lecannelier, 2001, 2002), como en sus aplicaciones. Se ha relacionado el apego con problemas de salud pública, tales como el maltrato infantil, el tipo de apego con los factores asociados a la resiliencia respecto de este tipo de maltrato (Aracena, Castillo, Haz, Muñoz, Román \& Bustos, 2000), la relación entre apego y trastornos alimentarios, entre ellos la anorexia (Cordella, 2002), la influencia del apego en la adaptación de niños adoptados (Rosas, Gallardo \& Angulo, 2000) y los patrones de apego en los niños/as institucionalizados (Méndez \& González, 2002). Sin embargo, es posible afirmar que la línea de investigación de apego en Chile es incipiente, puesto que se cuenta con escasas publicaciones en nuestro país.

\section{Conclusiones}

La presente sistematización invita a los investigadores a cuestionarse algunos temas teóricos y metodológicos de las intervenciones en apego como asimismo de los recursos económicos que cuentan los equipos de investigación en Chile para llevar a cabo investigación de calidad de acuerdo a los estándares internacionales (Flay et al. en Olds, 2007).

En primer lugar, es necesario cuestionarse ¿qué intervenir? Intervenir focalizándose sólo en el cambio en el estilo de apego, se hace difícil como constructo teórico. La mayoría de los efectos obtenidos en este tipo de intervención se pueden observar a largo plazo, y no necesariamente tienen un impacto directamente en la madre, sino en el desarrollo del hijo/a. En consecuencia, al momento de decidir qué evaluar, es relevante medir no sólo la variable resultado, en este caso el apego, sino también, incluir la medición de variables mediadoras, como la sensibilidad de las madres o 
cuidadores en distintos momentos. Así también, incluir la observación de efectos a mediano y largo plazo en el hijo/a, lo cual podría ser una consecuencia de una intervención de este tipo, no sólo en el ámbito afectivo sino en el desarrollo psicomotor, lenguaje, entre otros (Strasser, 2006).

En síntesis, se puede concluir que la literatura señala que entre los factores a considerar al momento de realizar intervenciones tempranas, los más relevantes son: el foco de la intervención, el tipo de riesgo familiar, la edad del niño/a al momento de la intervención y el porcentaje de desorganización de apego de la madre (Bakermans-Kraneburg, et al., 2005).

El foco más significativo es el aumento de sensibilidad de los padres (Bakermans-Kraneburg, et al., 2003). En consecuencia, si la variable apego no obtiene resultados positivos, es posible evaluar si en la variable mediadora -la sensibilidad de las madres-, hubo efectos. Acerca del tipo de riesgo familiar, la literatura señala que se ha trabajado en familias con problemas psicosociales y patologías, tales como: madres clínicamente deprimidas (Gelfland et al., 1996; Cooper \& Murray, 1997); díadas madre-hijo/as referidas clínicamente (Cohen et al., 1999), entre otros. Se ha intervenido además, en poblaciones con estresores contextuales múltiples, tales como; pobreza, monoparentalidad, bajo nivel educacional, bajo apoyo social percibido y madres con inadecuada parentalidad (Egeland \& Ericsson, 1993; Heinicke et al., 1999; Lyons-Ruth, Connell \& Grunenbaum, 1990).

En cuanto al diseño de la investigación, un punto relevante de prever es la mortalidad experimental. Es decir, que la muestra sea lo suficientemente grande como para soportar la pérdida de sujetos, y aún así poder dilucidar los efectos de la intervención (Gurung, 2006).

Teniendo en cuenta lo anterior, aparecen distintas interrogantes a responder al momento de diseñar una investigación relacionada con intervenciones efectivas para promover el apego seguro en la díada madre e hijo/a.

En segundo lugar, al momento de definir la población a intervenir, se deben considerar distintas variables. La literatura señala que la mayor efectividad se observa en grupos en riesgo, sin embargo, hay que controlar el tipo de riesgo al cual se está apuntando, para tener claridad acerca de los factores que están influyendo. Se ha visto que aquellos padres con psicopatologías graves, por ejemplo, no serían una buena población objetivo, ya que necesitan tener un nivel de salud mental adecuado para cumplir su rol de cuidadores (Bakermans-Kranenburg, van Izjerdoorn \& Juffer, 2005). Un desafío aun mayor es trabajar con la población general. Ammaniti et al. (2006) presenta uno de los primeros trabajos donde incorporan además de población en riesgo y clínica un grupo de la población general.

Finalmente, los instrumentos utilizados, deben ser válidos y confiables para permitir identificar las diferencias. Además, hay que considerar el contexto de aplicación, de manera que éste no interfiera con la medición.

Como investigadores chilenos es posible afirmar que el diseño de proyectos de investigación tendientes a evaluar la efectividad de programas de intervención temprana en apego supone la articulación de una serie de elementos teóricos, metodológicos y prácticos que preserven la sensibilidad de los diseños para capturar los efectos de dicha intervención. De lo contrario, la posibilidad de extraer conclusiones válidas de los estudios implementados se reduce ostensiblemente. La tarea para Chile presenta importantes desafíos, y requiere que los investigadores de esta área puedan unir esfuerzos tanto intelectuales como económicos en aras de generar conocimiento contextualizado con los recursos disponibles. 


\section{Referencias}

Ahern, K. \& R. Le Brocque (2005). Methodological Issues in the Effects of Attrition: Simple Solutions for Social Scientists. Field Methods, 17, 53-69.

Ainsworth, M.D.S., Blehar, M. C., Waters, E. \& Wall, S. (1978). Patterns of attachment: A psychological study of the strange situation. New Jersey: Erlbaum.

Ammaniti, M., Speranza, A., Tambelli, R., Muscetta, S., Lucarelli, L., Vismara, L., Odorisio, F. \& Cimino, F. (2006). A prevention and promotion intervention program in the field of motherinfant relationship. Infant Mental Health Journal, 27, 70-90.

Anisfeld, E., Casper, V., Nocyze, M. \& Cunningham, N. (1990). Does infant carrying promote attachment? An experimental study of the effects of increased physical contact on the development of attachment. Child Development. 61 (5), 1617-1627.

Aracena, M., Krause, M., Salvatierra, L., Soto, M., Pantoja, T., Farah, C. \& Méndez, M. J. A. (2007). Cost-Effectiveness evaluation of home visit program for adolescent mothers. Manuscrito enviado para publicación.

Bakermans-Kranenburg, M., Juffer, F. \& van Izendoorn, M. (1998). Interventions with video feedback and attachment discussions: Does type of maternal insecurity make a difference? Infant Mental Health Journal, 19 (2), Special issue: 6th World Congress, World Association of Infant Mental Health. 202-219.

Bakermans-Kranenburg, M., van Izendoorn, M. \& Juffer, F. (2005). Disorganized Infant Attachment and preventive interventions: A review and meta-analysis. Infant Mental Health Journal, 26 (3), 191-216.

Bakermans-Kranenburg, M., Juffer, F., Van IJzendoorn, M. (2003). Less is More: Meta-analyses of sensitivity and attachment interventions in early childhood. Psychological Bulletin, 129 (2), 195-215.

Bedregal (2006). Eficacia y efectividad en la atención de niños entre 0 y 4 años [Versión electrónica] Expansiva , 79, 1-30.

Belsky, J. (1999). Interactional and contextual determinants of attachment security. En J. Cassidy, \& P. Shaver. Handbook of attachment: theory, research and clinical applications (pp. 249-265). New York: Guilford Press.

Cohen, N., Muir, E., Lojkasek, M., Muir, R., Parker, C., Barwick, M. \& Brown, M. (1999). Watch, wait and wonder: testing the effectiveness of a new approach to mother- infant psychotherapy. Infant Mental Health Journal. 20 (4), 429-451.

Cooper, P.J., \& Murray, L. (1997). The impact of psychological treatments of postpartum depression on maternal mood and infant development. En L. Murray \& P. J. Cooper, Postpartum depression and child development (pp. 201-261). New York: Guilford Press.

Crittenden, P. M. (1985). Social networks, Quality of Child Rearing, and Child Development. Child Development, 56, 1299-1313.

Crnic, K. A. \& Greenberg, M. T. (1987). Maternal stress, social support, and coping: influences on the early mother-infant relationship. En C. F. Z. Boukydis (Ed.). Research on support for parents and infants in tha postnatal period (pp. 25-40). Norwood, NJ: Ablex.

Egeland, B. \& Erickson, M.F. (1993). Attachment theory and findings: Implications for prevention and intervention. En S. Kramer \& H. Parens (Eds.). Prevention in mental health: Now, tomorrow, ever? (pp. 21-50). Northvale, NJ: Jason Aronson.

Garrido, L., Santelices, M. P., Pierrehumbert, P., Armijo, I. (2007). Validación chilena del cuestionario de evaluación de apego en el adulto CaMir. (Artículo enviado a la Revista Latinoamericana de Psicología). 
Gelfland, D., Teti, D., Seiner, S. \& Jameson, P. (1996). Helping mothers fight depression: Evaluation of a home-based intervention program for depressed mothers and their infants. Journal of Clinical Child Psychology, 25, 406-422.

Gurung, R. (2006). Models of Behavior Change. En M. Sordi (Ed.), Health Psychology, A cultural Approach (pp. 172-199). Thomson Wadswoth: Canada.

Heinicke, C.M., Diskin, S.D., Ramsey-Klee, D.M. \& Oates, D.S. (1986). Pre and postbirth antecedents of 2-year-old attention, capacity for relationships, and verbal expressiveness. Developmental Psychology, 22 (6), 777-787.

Heinicke, C.M., Beckwith, L. \& Thompson, A. (1988). Early intervention in the family system: A framework and review. Infant Mental Health Journal, 9 (2), 111-141.

Heinicke, C.M., Fineman, N. R., Ruth, G., Recchia, S.L., Guthrie, D. \& Rodning, C. (1999). Relationship-based intervention with at-risk mothers: outcome in the first year of life. Infant Mental Health Journal, 20, 349-374.

Heinicke, C. M., Fineman, N.R., Ponce, V.A. \& Guthrie, D. (2001). Relation-Based intervention with at-risk mothers: outcomes in the second year of life. Infant Mental Health Journal, 22, 431-462.

Huth-Bocks, A., Levendosky, A., Bogat, A. \& von Eye, A. (2004). The Impact of Maternal Characteristics anda Contextual Variables on Infant-Mother Attachment. Child Development. 75 (2), 480-496.

Huxley, P. \& Warner, R. (1993) Primary prevention of parenting dysfunction in high-risk cases. American Journal of Orthopsychiatry, 63, 582-588.

Jacobson, S., Frye, K. (1991) Effect of maternal social support on attachment: Experimental evidence. Child Development . 62 (3) 572-582.

Juffer, F., Bakermans-Kranenburg, M. \& van Izendoorn, M. (2005). The importance of Parenting in the development of disorganized attachment: evidence of a preventive intervention study in adoptive families. Journal of Child Psychology and Psychiatry, 46, 3, 263-274.

Lyons-Ruth, K., Connell, D., Grunebaum, H. (1990). Infants at social risk: Maternal depression and family support services as mediators of infant development and security of attachment. Child Development, 61, 85-98.

Madigan, S., Bakermans-Kranenburg, M., van Izendoorn, M., Moran, G., Pederson, D. \& Benoit, D. (2006). Unresolved states of mind, anomalous parental behavior, and disorganized attachment: A review and meta-analysis of a transmission gap. Attachment \& Human Development, 8, 89-111.

Olds, D.L., Henderson, C.R., Chamberlin, R., \& Tatelbaum, R. (1986). Preventing child abuse and neglect: A randomized trial of nurse home visitation. Pediatrics, 78, 65-77.

Olds, D., Robinson, J., O’Brien, R. Luckey, D., Pettitt, L., Henderson, C. et al. (2002). Home visiting by paraprofessionals and by nurses: a randomized, controlled trial. Pediatrics, 110, 486-496.

Olds, D., Robinson, J., Pettit, L., Luckey, D., Holmberg, J., Rossana K. et al. (2004). Effects of home visits by paraprofessionals and by nurses: age 4 follow-up results of a randomized trial. Pediatrics, 114 (6), 1560-1568.

Olds, D., Sadler S. \& Kitzman H. (2007). Programs for parents of infants and toddlers: recent evidence from randomized trials. Journal of Child Psychology and Psychiatry 48 (3/4), 355-391.

Ortiz, J., Borré, A., Carrillo, S. \& Gutiérrez, G. (2006). Relación de apego en madres adolescentes y sus bebés canguro [Versión electrónica]. Revista Latinoamericana de Psicología, 38 (1), $71-$ 86. 
Organización de las Naciones Unidas (2000). Juventud, Población y Desarrollo en América Latina y el Caribe [Youth, population and development in Latin America and the Caribbean]. México, D.F.: CEPAL.

Pierrumbert, B., Karmaniola, A., Sieye, A., Meister, C., Miljkovitch, R. \& Halfon, O. (1996). Les modèles de relations: Développement d'un autoquestionnaire d'attachment pour adultes, CaMir. Psyquiatrie de l'enfant, vol. XXXIX, 1, 161-206.

Ramey, S. L. \& Ramey, C. T. (2006). Early Educational Interventions: Principles of Effective and Sustained Benefits from Targeret Early Educations Programs. En D. K. Dickinson \& S. B. Neuman (Eds). Handbook of Early Literacy Research, Vol. 2. Guilford Press: New York.

Santelices, M.P., Ramírez, V., Armijo, I., Pérez-Salas C.P. (2008). Propiedades psicométricas de la versión chilena del cuestionario de apego en adultos “CAMIR". Psicopatología y Salud Mental, 11, 49-59.

Sajaniemi, N., Makela, J., Salokorpi, T. (2001). Cognitive performance and attachment patterns at four years of age in extremely low birth weight infants after early intervention. European Child \& Adolescent Psyvhiatry. 10, 120-19.

Strasser, K. (2006). Evaluación de programas de intervención temprana [Versión electrónica] Expansiva, 78, 1-26.

Streiner, D. \& Norman, G. 1999). Health Measurment scales. A practical guide to their development and use.Oxford: Oxford University Press.

Van den Boom, D. (1994). The influence of temperament and mothering on attachment exploration: An experimental manipulation of sensitive responsiveness among lower-class mothers with irritable infants. Child Development, 65, 1457-1477.

van Izendoorn, M. (1995). Adult attachment representations, parental responsiveness, and infant attachment. Psychological Bulletin, 117(3), 387-403.

Velderman, K., Bakermans-Kranenburg, M., Juffer, F. \& van Izendroorn, M. (2006). Effects of Attachment-Based Interventions on Maternal Sensitivity ans Infant Attachment: Differential susceptibility of Highly Reactive Infants. Journal of Family Psychology, 20 (2), 266-274.

Whitehurst, D. S., Arnold, J. N., Epstein, A. L. Angell, M. Smith \& J. E. Fischel (1994). A picture Book Reading Intervention in Day Care and Home for children from Low-Income Families. Development Pshychology, 30 (5), 679-696.

Wutzke, S.; Conigrave, K.; Kogler, B.; Saunders, J. \& Hall, W. (2000). Drug and Alcohol Review, 19 (2), 159- 163. 